\title{
Impurity Modulated Static Linear and First Nonlinear Polarizabilities of Doped Quantum Dots
}

\author{
Nirmal Kr Datta ${ }^{1}$ and Manas Ghosh ${ }^{2}$ \\ ${ }^{1}$ Department of Physics, Suri Vidyasagar College, Suri, Birbhum 731101, West Bengal, India \\ ${ }^{2}$ Department of Chemistry, Physical Chemistry Section, Visva Bharati University, Santiniketan, \\ Birbhum 731235, West Bengal, India \\ Correspondence should be addressed to Manas Ghosh, pcmg77@rediffmail.com \\ Received 7 January 2012; Accepted 26 February 2012 \\ Academic Editors: G. Bellanca, D. Cojoc, E. Lidorikis, and G. Ma
}

Copyright (C) 2012 N. K. Datta and M. Ghosh. This is an open access article distributed under the Creative Commons Attribution License, which permits unrestricted use, distribution, and reproduction in any medium, provided the original work is properly cited.

We explore the pattern of linear and first nonlinear optical (NLO) response of repulsive impurity doped quantum dots harmonically confined in two dimensions. The dopant impurity potential chosen assumes Gaussian form. The quantum dot is subject to a static electric field. For some fixed values of transverse magnetic field strength $\left(\omega_{c}\right)$, and harmonic confinement potential $\left(\omega_{0}\right)$, the influence of impurity strength $\left(V_{0}\right)$, impurity stretch $(\xi)$, and impurity location $\left(r_{0}\right)$ on the diagonal components of static linear $\left(\alpha_{x x}\right.$ and $\left.\alpha_{y y}\right)$, and the first NLO $\left(\beta_{x x x}\right.$ and $\left.\beta_{y y y}\right)$ responses of the dot are computed through linear variational route. The investigation reveals the crucial roles played by the various impurity parameters in modulating these optical responses. Interestingly, maximization in the first NLO responses has been observed for some particular dopant location and at some particular value of spatial stretch of the dopant.

\section{Introduction}

The study of impurity states in low-dimensional heterostructures has emerged as an important aspect to which many theoretical and experimental works have been dedicated. As a result, nowadays the researches on doped semiconductor devices come out to be ubiquitous [1]. The doped system has quantized properties making them ideal objects for scientific study and robust applications. The dopants modify the chemical potential of a material. The control of optoelectronic properties of a wide range of semiconductor devices [2-4] now turns out to be the most fascinating aspect of impurity doping in such materials.

Miniaturization of semiconductor devices reaches the bottom of the avenue with the advent of so-called lowdimensional structures such as quantum dots (QDs), with QD's new perspectives and delicacies in the field of impurity doping emerge, due to the mingling of new confinement sources with impurity related potentials [5]. Such confinement, coupled with the dopant location, can dramatically alter the electronic and optical properties of the system $[6,7]$.
For quite sometimes, search for molecules and materials with high linear and nonlinear susceptibilities and ultrafast response time has been pursued all over the world [8-14]. The targeted molecules are important in communication technology, data storage, optical switching, and so forth $[9,11,15]$. As a result of numerous studies, certain broad designing clues have emerged. Based on these guidelines, most often one tries to design donor-acceptor molecules with large charge transfer from the donor to the acceptor moiety. NLO response is maximized for certain optimal combinations of charge transfer and hopping interactions and the length over which the charge is transferred [16-19]. Modulation in the molecular electronic parameters brought through subtle structural changes or change in substitution changes the electronic wave function and therefore the electron density distribution which ultimately shapes all the properties of atoms and molecules. It would be crucial to have at one's disposal, systems, the electronic structures of which could be continuously tuned by adjusting suitable control parameters. In molecules, these variations are only discrete, having caused by either a change of substituent 
or by structural alteration or by both. Quantum dots are representatives of the systems where the electronic structural dispositions can be practically continuously varied as functions of a few of the system parameters. From the above discussion, it appears inevitable that variation in the electronic structure of the dot induced by the impurity could subtly shape all its properties. It is thus crucial to study the role of dopant impurity on the optical properties of the quantum dots. A thorough expedition through literature reveals some notable works on the optical properties of impurity doped quantum dots [20, 21]. Doped quantum dots, thus, beyond any doubt, possess the potential to exhibit high linear and nonlinear optical response properties and would thus be principally applied in the area of alloptical signal processing. A detailed investigation of various dopant parameters relevant to optical signal processing would continue to be a topic of active research. In what follows, of late, we have also studied the frequency dependent linear and nonlinear polarizabilities of doped quantum dots as functions of various dopant parameters [22, 23].

In the present manuscript, we inspect the diagonal components of linear $\left(\alpha_{x x}\right.$ and $\left.\alpha_{y y}\right)$ and first nonlinear polarizabilities $\left(\beta_{x x x}\right.$ and $\left.\beta_{y y y}\right)$ of a repulsive impurity doped quantum dot subject to a static electric field. Following earlier works on the effects of a repulsive scatterer in multicarrier dots in the presence of magnetic field [24, 25], here we have considered that the QD is doped with a repulsive Gaussian impurity. When the impurity is doped in an on-center location, it does not destroy the inversion symmetry of the dot and consequently the emergence of $\beta$ value is ruled out. However, nonzero $\alpha$ values are envisaged. At off-center dopant locations, however, emergence of both nonzero $\alpha$ and $\beta$ values is observed owing to the destruction of inversion symmetry. We have found that, in conjunction with the dopant location, the strength and the spatial stretch of the dopant also affect the polarizability values quite prominently.

\section{Method}

We consider the energy eigenstates of an electron subject to a harmonic confinement potential $V(x, y)$ and a perpendicular magnetic field $B$, where $V(x, y)=(1 / 2) m^{*} \omega_{0}^{2}\left(x^{2}+y^{2}\right)$, $\omega_{c}=e B / m^{*} c$, and Landau gauge $[A=(B y, 0,0)]$ have been used. $\omega_{0}, \omega_{c}$, and $A$ stand for harmonic confinement potential, cyclotron frequency (a measure of magnetic confinement offered by $B$ ), and vector potential, respectively. The Hamiltonian in our problem reads

$$
\begin{aligned}
H_{0}^{\prime}= & -\frac{\hbar^{2}}{2 m^{*}}\left(\frac{\partial^{2}}{\partial x^{2}}+\frac{\partial^{2}}{\partial y^{2}}\right)+\frac{1}{2} m^{*} \omega_{0}^{2} x^{2}, \\
& +\frac{1}{2} m^{*}\left(\omega_{0}^{2}+\omega_{c}^{2}\right) y^{2}-i \hbar \omega_{c} y \frac{\partial}{\partial x} .
\end{aligned}
$$

Define $\Omega^{2}=\omega_{0}^{2}+\omega_{c}^{2}$ as the effective frequency in the $y$-direction. In real QDs, the electrons are confined in 3 dimensions that is, the carriers are dynamically confined to zero dimensions. The confinement length scales $R^{1}, R^{2}$, and $R^{3}$ can be different in three spatial directions, but typically
$R^{3} \ll R^{1} \simeq R^{2} \simeq 100 \mathrm{~nm}$. In models of such dots, $R^{3}$ is often taken to be strictly zero and the confinement in the other two directions is described by a potential $V$ with $V(x) \rightarrow \infty$ for $|x| \rightarrow \infty, x=\left(x^{1}, x^{2}\right) \in R^{2}$. A parabolic potential, $V=(1 / 2) \omega|x|^{2}$, is often used as a realistic and at the same time computationally convenient approximation. Assuming that the $z$-extension could be effectively considered zero, the electronic properties in these nanostructures have been successfully described within the model of the 1-electron motion in 2-d harmonic oscillator potential in the presence of a magnetic field [26-28]. Now, intrusion of impurity perturbation transforms the Hamiltonian to

$$
H_{0}\left(x, y, \omega_{c}, \omega_{0}\right)=H_{0}^{\prime}\left(x, y, \omega_{c}, \omega_{0}\right)+V_{\mathrm{imp}}\left(x_{0}, y_{0}\right),
$$

where $V_{\text {imp }}\left(x_{0}, y_{0}\right)=V_{\text {imp }}(0)=V_{0} e^{-\xi\left[\left(x-x_{0}\right)^{2}+\left(y-y_{0}\right)^{2}\right]}$ with $\xi>0$ and $V_{0}>0$ for repulsive impurity, and $\left(x_{0}, y_{0}\right)$ denotes the position of the impurity center. In QD's, the electrons visit an almost equipotential surface wherein they are set free. These electrons are carrier electrons. In reality, regular dot occurs rarely as the deformation of the boundary of QD's modifies the effective potential of the carrier electrons to bunch of uneven pockets. Sometimes, these electrons play the role of defects themselves (repulsive impurity) [29]. $V_{0}$ is a measure of the strength of impurity potential whereas $\xi$ determines the extent of influence of the impurity potential. A large value of $\xi$ indicates that the spatial extension of impurity potential is highly restricted whereas a small $\xi$ accounts for spatially diffused one. Thus, a change in $\xi$ in turn causes a change in the extent of dot-impurity overlap that affects the excitation pattern noticeably $[22,23]$. The presence of repulsive scatterer simulates dopant with excess electrons. The choice of Gaussian impurity potential is not arbitrary as it has been exploited by several investigators [3035].

We write the trial wave function $\psi(x, y)$ as a superposition of the product of harmonic oscillator eigenfunctions $\phi_{n}(\alpha x)$ and $\phi_{m}(\beta y)$, respectively, as follows:

$$
\psi(x, y)=\sum_{n, m} C_{n, m} \phi_{n}(\alpha x) \phi_{m}(\beta y),
$$

where $C_{n, m}$ are the variational parameters and $\alpha=\sqrt{m^{*} \omega_{0} / \hbar}$ and $\beta=\sqrt{m^{*} \Omega / \hbar}$. The matrix elements of $V_{\text {imp }}$ are given by

$$
\begin{aligned}
& \left(V_{\text {imp }}\right)_{n, m ; n^{\prime}, m^{\prime}} \\
& \quad=V_{0}\left\langle\phi_{n}(\alpha x) \phi_{m}(\beta y)\left|e^{-\xi\left[\left(x-x_{0}\right)^{2}+\left(y-y_{0}\right)^{2}\right]}\right| \phi_{n^{\prime}}(\alpha x) \phi_{m^{\prime}}(\beta y)\right\rangle \\
& \quad=V_{0} I_{1} I_{2},
\end{aligned}
$$

where

$$
\begin{aligned}
I_{1} & =\left\langle\phi_{n}(\alpha x)\left|e^{-\xi\left(x-x_{0}\right)^{2}}\right| \phi_{n}^{\prime}(\alpha x)\right\rangle \\
& =A \int_{-\infty}^{+\infty} H_{n}(\alpha x) H_{n^{\prime}}(\alpha x) e^{-\alpha^{2} x^{2}} e^{-\xi\left(x-x_{0}\right)^{2}} d x, \\
I_{2} & =\left\langle\phi_{m}(\beta y)\left|e^{-\xi\left(y-y_{0}\right)^{2}}\right| \phi_{m}^{\prime}(\beta y)\right\rangle \\
& =B \int_{-\infty}^{+\infty} H_{m}(\beta y) H_{m^{\prime}}(\beta y) e^{-\beta^{2} y^{2}} e^{-\xi\left(y-y_{0}\right)^{2}} d y,
\end{aligned}
$$


with $A=\alpha /\left(2^{n+n^{\prime}} n ! n^{\prime} ! \pi\right)^{1 / 2}$ and $B=\beta /\left(2^{m+m^{\prime}} m ! m^{\prime} ! \pi\right)^{1 / 2}$. With the transformations $\delta_{1}^{2}=\alpha^{2}+\xi, \delta_{2}^{2}=\beta^{2}+\xi, \lambda_{1}=$ $\exp \left[-\left(\xi x_{0}^{2}\left(\delta_{1}^{2}-\xi\right)\right) / \delta_{1}^{2}\right]$, and $\lambda_{2}=\exp \left[-\left(\xi y_{0}^{2}\left(\delta_{2}^{2}-\xi\right)\right) / \delta_{2}^{2}\right]$, one can write

$$
\begin{aligned}
& I_{1}=A \lambda_{1} \int_{-\infty}^{+\infty} H_{n}\left(\alpha^{*} u\right) H_{n^{\prime}}\left(\alpha^{*} u\right) e^{-\left(u-\rho_{1}\right)^{2}} d u, \\
& I_{2}=B \lambda_{2} \int_{-\infty}^{+\infty} H_{m}\left(\beta^{*} v\right) H_{m^{\prime}}\left(\beta^{*} v\right) e^{-\left(v-\rho_{2}\right)^{2}} d v,
\end{aligned}
$$

where, $\rho_{1}=\xi x_{0} / \delta_{1}, \rho_{2}=\xi y_{0} / \delta_{2}, \alpha^{*}=\alpha / \delta_{1}, \beta^{*}=\beta / \delta_{2}$, $u=\delta_{1} x$, and $v=\delta_{2} y$. With the help of the standard integral [36], it is now easy to write

$$
\begin{gathered}
I_{1}=D_{1} \sum_{k=0}^{\min \left(n, n^{\prime}\right)} f\left(k, n, n^{\prime}\right), \\
I_{2}=D_{2} \sum_{l=0}^{\min \left(m, m^{\prime}\right)} g\left(l, m, m^{\prime}\right),
\end{gathered}
$$

where

$$
\begin{aligned}
f\left(k, n, n^{\prime}\right)= & 2^{k} \cdot k ! \cdot{ }^{n} C_{k} \cdot{ }^{n^{\prime}} C_{k} \cdot\left(1-\alpha^{* 2}\right)^{\left(n+n^{\prime}\right) / 2-k} \\
& \cdot H_{n+n^{\prime}-2 k}\left(\alpha_{1} \rho_{1}\right) \\
g\left(l, m, m^{\prime}\right)= & 2^{l} \cdot l ! \cdot{ }^{m} C_{l} \cdot{ }^{m^{\prime}} C_{l} \cdot\left(1-\beta^{* 2}\right)^{\left(m+m^{\prime}\right) / 2-l} \\
& \cdot H_{m+m^{\prime}-2 l}\left(\beta_{1} \rho_{2}\right),
\end{aligned}
$$

with $D_{1}=A \lambda_{1} \pi^{1 / 2} / \delta_{1}$ and $D_{2}=B \lambda_{2} \pi^{1 / 2} / \delta_{2}$. Thus, finally, we obtain

$$
\begin{aligned}
\left(V_{\mathrm{imp}}\right)_{n, m ; n^{\prime}, m^{\prime}}= & V_{0} \cdot D_{1} \cdot D_{2} \\
& \cdot \sum_{k=0}^{\min \left(n, n^{\prime}\right)} \sum_{l=0}^{\min \left(m, m^{\prime}\right)} f\left(k, n, n^{\prime}\right) \cdot g\left(l, m, m^{\prime}\right) .
\end{aligned}
$$

$H_{n}(x)$ stands for the Hermite polynomials of $n$th order. The $p$ th eigenstate of the system in this representation can be written as

$$
\psi_{p}(x, y)=\sum_{i j} C_{i j, p}\left\{\phi_{i}(\alpha x) \phi_{j}(\beta y)\right\},
$$

where $i, j$ are the appropriate quantum numbers, respectively, and $(i j)$ are composite indices specifying the direct product basis.

In presence of a static electric field of strength $\varepsilon$, the effective Hamiltonian of the system $\left(H_{\text {eff }}\right)$ becomes

$$
H_{\text {eff }}=H_{0}-\left\{\varepsilon_{x}|e| x+\varepsilon_{y}|e| y\right\} .
$$

We have determined the values of $\left\langle E\left( \pm \varepsilon_{x}, \varepsilon_{y}=0\right)\right\rangle$, $\left\langle E\left( \pm 2 \varepsilon_{x}, \varepsilon_{y}=0\right)\right\rangle,\left\langle E\left(\varepsilon_{x}=0, \pm \varepsilon_{y}\right)\right\rangle$, and $\left\langle E\left(\varepsilon_{x}=0, \pm 2 \varepsilon_{y}\right)\right\rangle$ and we used the data to compute the direct components of polarizabilities of the dot by the following relations obtained by numerical differentiation [37-41]:

$$
\begin{aligned}
\alpha_{x x} \varepsilon_{x}^{2}= & \frac{5}{2}\langle E(0)\rangle-\frac{4}{3}\left[\left\langle E\left(\varepsilon_{x}\right)\right\rangle+\left\langle E\left(-\varepsilon_{x}\right)\right\rangle\right] \\
& +\frac{1}{12}\left[\left\langle E\left(2 \varepsilon_{x}\right)\right\rangle+\left\langle E\left(-2 \varepsilon_{x}\right)\right\rangle\right],
\end{aligned}
$$

TABle 1: The width of the impurity domain in $\mathrm{nm}$ for different $\xi$ values.

\begin{tabular}{lc}
\hline$\xi$ (a.u.) & $d(\mathrm{~nm})$ \\
\hline 0.00001 & 14.07 \\
0.0001 & 4.45 \\
0.001 & 1.41 \\
0.01 & 0.44 \\
0.1 & 0.14 \\
\hline
\end{tabular}

and a similar expression for $\alpha_{y y} \varepsilon_{y}^{2}$ :

$$
\begin{aligned}
\beta_{x x x} \varepsilon_{x}^{3}= & {\left[\left\langle E\left(\varepsilon_{x}, 0\right)\right\rangle-\left\langle E\left(-\varepsilon_{x}, 0\right)\right\rangle\right] } \\
& -\frac{1}{2}\left[\left\langle E\left(2 \varepsilon_{x}, 0\right)\right\rangle-\left\langle E\left(-2 \varepsilon_{x}, 0\right)\right\rangle\right],
\end{aligned}
$$

and a similar expression is used for computing $\beta_{y y y}$ component. In these expressions, $\left\langle E\left( \pm \epsilon_{x}\right)\right\rangle$ represents average energy of the system when $\varepsilon_{y}=0$ and $\varepsilon_{x} \neq 0$. Analogously, we have also written $\left\langle E\left( \pm \epsilon_{y}\right)\right\rangle$. The above relations indicate different combinations of electric field intensities and orientations.

\section{Results and Discussion}

3.1. System Parameters. The model Hamiltonian (cf. (1)) can be made to represent a 2-d quantum dot with a single carrier electron $[28,42]$. The form of the confinement potential indicates lateral electrostatic confinement of the electrons in the $x-y$ plane. Thus, it is disklike which is normally fabricated by molecular beam epitaxy (MBE) technique. Because of unequal confinements in $x$ and $y$-directions, the symmetry is nonhomogeneous. $m^{*}$ is the effective electronic mass appropriate for describing the motion of the electrons within the lattice of the material to be used. We have used $m^{*}=0.5 m_{0}$ and set $\hbar=e=m_{0}=a_{0}=1$. The radial position of dopant $\left(r_{0}\right)$ has been varied from 0.0 a.u. (on-center) to 70.71 a.u. (off-center) positions. In the linear variational calculation, we have used basis functions (cf. (3)) with $n, m=0-20$ for each of the directions $(x, y)$. The direct product basis spans a space of $(21 \times 21)$ dimension. We have checked that the basis functions span the 2-d space effectively completely, at least with respect to representing the observables under investigation. We have made the convergence test with still greater number of basis functions.

We have made some attempt to reasonably connect our theoretical parameters to the real life-doped QD. The parameter $\xi$ in the impurity potential can be correlated to $1 / d^{2}$, where $d$ is proportional to the width of the impurity potential $[24,25]$. Table 1 gives the $d$ values in $\mathrm{nm}$ corresponding to different $\xi$ values to have a feelings of the actual extension of the impurity domain. The $m^{*}$ value that we have used $\left(m^{*}=0.5\right.$ a.u. $)$ closely resembles Ge quantum dots $\left(m^{*}=0.55\right.$ a.u. $)$. The maximum value of the dopant strength $\left(V_{0}\right)$ was limited to $\sim 10^{-3}$ a.u. or $27.2 \mathrm{meV}$ and the applied magnetic field is of the order of milliTesla (mT). Here, we want to mention that our method is not strictly a perturbation theory in that sense where 
some perturbation is added to the original Hamiltonian through the agency of a perturbation parameter. In our work, we have exploited linear variation principle to determine the approximate eigenstates of the system as it was not possible to solve the time-independent Schrodinger equation containing impurity in the Hamiltonian as well as in the presence of static electric field. Thus, firstly, we have evaluated the approximate eigenstates of the system taking harmonic oscillator eigenfunctions as the basis function. This involves modulation of the linear combination coefficients. Obviously, the modulation was not arbitrary but was governed by the diagonalization of the Hamiltonian matrix containing the contributions from impurity potential and static electric field. Thus, the method is actually a basis function expansion technique with properly adjusted linear combination coefficients. The validity of the method lies in the fact that how far it can endure the increase in $V_{0}$ values so that normalization is not hampered. We did not make any forceful normalization of the wave function and checked that inherent normalization is well maintained even with a $V_{0}$ value as large as $1.0 \times 10^{-3}$ a.u. for a very large basis size $(21 \times 21)$ indicating that our method is quite robust so far as stability of the method is concerned. The static electric field has an intensity of $2.57 \times 10^{4} \mathrm{Vm}^{-1}$. We believe that these values might give some realization of real systems containing actual impurity.

\subsection{Aspects of Polarizability Components}

3.2.1. Influence of Impurity Location. Figure 1 depicts the variation of components of linear polarizability $\left(\alpha_{x x}\right.$ and $\left.\alpha_{y y}\right)$ in $x$ and $y$ directions as functions of radial position $\left(r_{0}\right)$ of the dopant. Both the components are found to decrease monotonically with increase in $r_{0}$. An increase in $r_{0}$ takes the dopant away from the dot confinement and consequently there occurs a reduction in dot-impurity overlap. As a result, the extent of repulsive interaction also diminishes which is reflected in the observed behaviors of linear polarizability components. From the plot, it has also been observed that the $\alpha_{y y}$ value is lower than $\alpha_{x x}$ component. This could be due to varied extent of confinement in $x$ and $y$ directions. A close look at (1) reveals that the effective harmonic frequency along $y$-axis is $\Omega=\sqrt{\omega_{0}^{2}+\omega_{c}^{2}}$ while that along $x$-axis is $\omega_{0}$. Thus, the $y$-direction is under stronger confinement in comparison to the $x$-direction and naturally enjoys less dispersive character. Consequently, the linear polarizability components which are intimately connected with the dispersive nature of the system assume different values in $x$ and $y$ directions. It needs to be mentioned that macroscopic polarizability should be isotropic for a symmetric QD in a perpendicular magnetic field. In our investigation, we have found anisotropy in the values of $\alpha_{x x}$ and $\alpha_{y y}$ on a microscopic scale because of a nonzero $\omega_{c}$. We feel that on a macroscopic scale, the anisotropies arising out of various microscopic components may balance each other leading to no net anisotropy of the system. However, a more extensive and exhaustive computation is required for an in depth investigation.

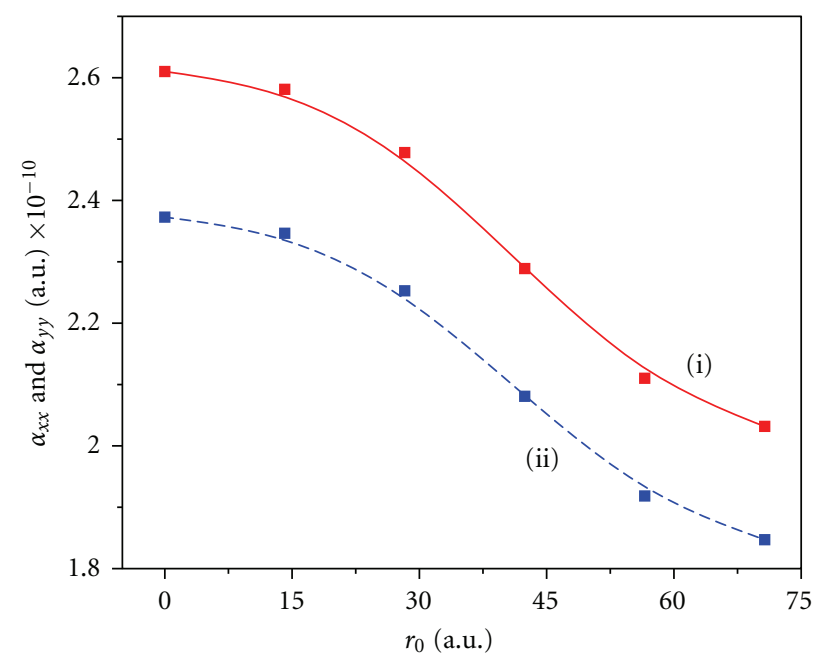

Figure 1: Plots of $\alpha_{x x}$ and $\alpha_{y y}$ versus $r_{0}$ with $V_{0}=1.0 \times 10^{-6}$ a.u. and $\xi=0.001$ a.u. (i) for $\alpha_{x x}$ and (ii) for $\alpha_{y y}$.

We now investigate the diagonal components of first hyperpolarizability $\left(\beta_{x x x}\right.$ and $\left.\beta_{y y y}\right)$ of the doped dot. For the emergence of nonzero $(\beta)$, doping of impurity at offcenter position is essential. An impurity doping at oncenter position does not destroy the inversion symmetry of the Hamiltonian and is therefore unable to generate nonzero $\beta$ value. Both the $\beta$ components exhibit maximization at a particular dopant location as the impurity is shifted away from the dot center (Figure 2). A shift of the dopant away from the dot confinement center is endowed with opposite consequences. On one hand, the intensity of dot confinement certainly decreases thereby favoring the high optical response. On the other hand, the said shift also decreases the extent of dot-impurity repulsive interaction and discourages emergence of high optical response. So far as generation of $\beta$ values are concerned; it appears that the interplay of these two opposing factors are responsible for the aforesaid maximization. For high NLO response, therefore, the designer quantum dots appear to be not hard to find. The requirement being the controlled incorporation of the dopant at definite site to break the symmetry of the confinement potential. In this regard, we should mention that of late there are some excellent experiments which show the mechanism of dopant incorporation [43] and how such incorporations can be controlled [44].

3.2.2. Influence of Impurity Strength. We have now varied $V_{0}$ over a wide range to observe its influence on the polarizability components. Figure 3 exhibits the $\alpha_{x x}$ and $\alpha_{y y}$ profiles as function of dopant strength for on and two offcenter $\left(r_{0}=42.43\right.$ a.u. and 70.71 a.u.) dopant locations. The plots reveal how a variation in the dopant strength can influence the polarizability components. We notice that at all dopant locations both the linear polarizability components exhibit a saturation at high dopant strength. Within extremely small dopant strength regime $\left(V_{0} \rightarrow 0\right)$, there occurs an initial uprise in the polarizability values with 


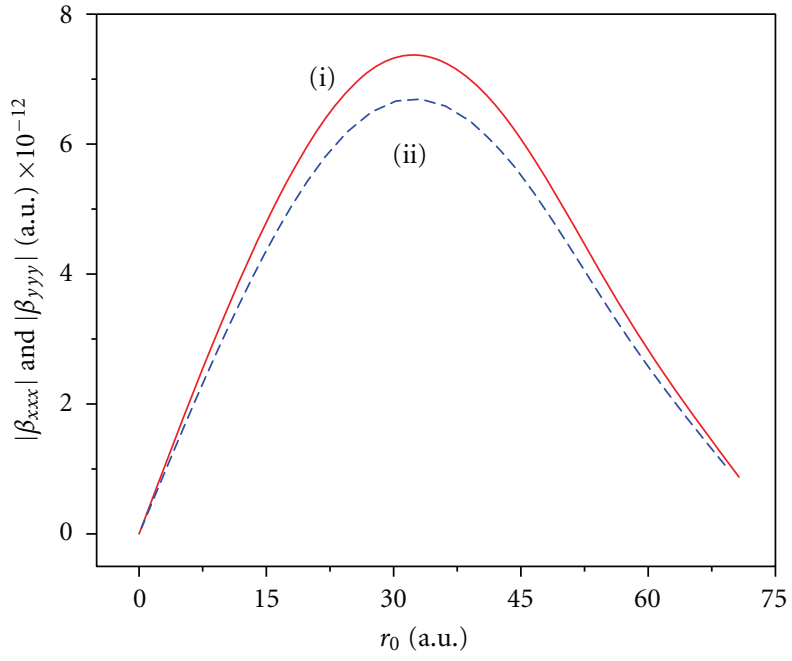

Figure 2: Plots of $\left|\beta_{x x x}\right|$ and $\left|\beta_{y y y}\right|$ versus $r_{0}$ with $V_{0}=1.0 \times$ $10^{-6}$ a.u. and $\xi=0.001$ a.u. (i) for $\left|\beta_{x x x}\right|$ and (ii) for $\left|\beta_{y y y}\right|$.

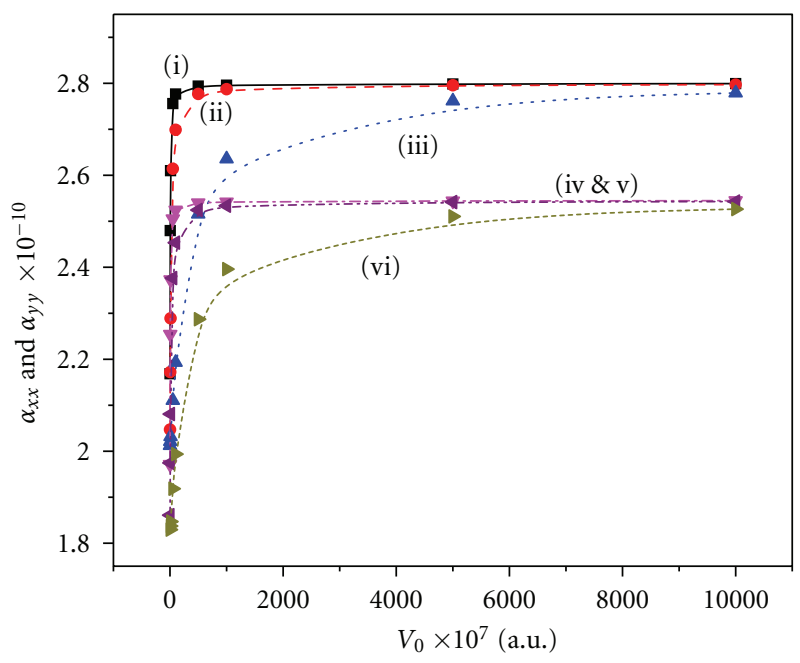

FIGURE 3: Plots of $\alpha_{x x}$ and $\alpha_{y y}$ versus $V_{0}$ with $\xi=0.001$ a.u. at on and two off-center dopant locations: (i) for $\alpha_{x x}$ at $r_{0}=0.0$ a.u., (ii) for $\alpha_{x x}$ at $r_{0}=42.43$ a.u., (iii) for $\alpha_{x x}$ at $r_{0}=70.71$ a.u., (iv) for $\alpha_{y y}$ at $r_{0}=0.0$ a.u., (v) for $\alpha_{y y}$ at $r_{0}=42.43$ a.u., and (vi) for $\alpha_{y y}$ at $r_{0}=70.71$ a.u.

increase in $V_{0}$. It seems that in this regime the dot-impurity interaction is very feeble. Small increase in dopant strength in this domain primarily causes some sort of development in the dot-impurity repulsive interaction and we observe an initial surge in the polarizability values. At large $V_{0}$ the plots show some kind of saturation indicating a steady extent of dot-impurity interaction. As observed earlier now also we find that $\alpha_{y y}$ values are somewhat lower in magnitude than $\alpha_{x x}$ values. Moreover, from the plots, we find that while the values of a particular component for on and near off-center dopant locations $\left(r_{0}=42.43\right.$ a.u. $)$ are in close proximity, the value drops substantially at far off-center

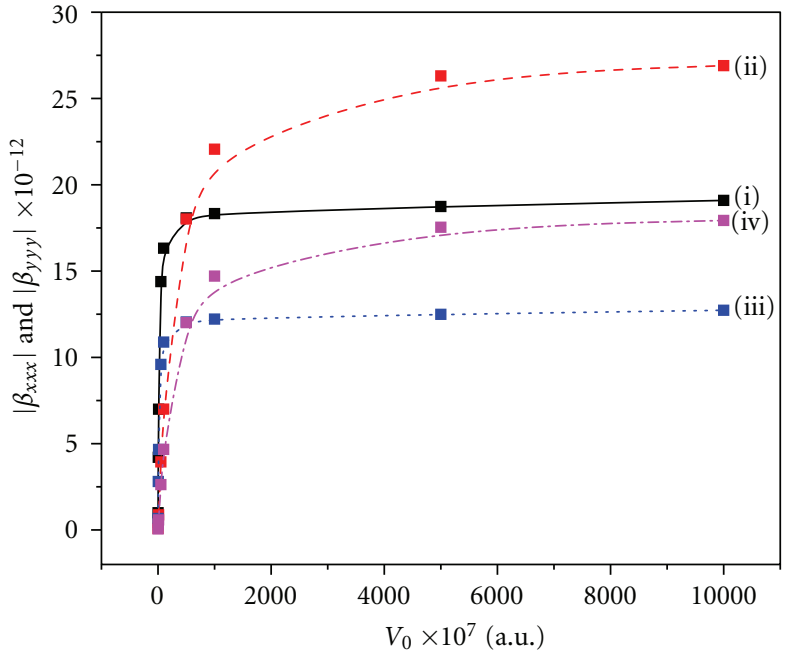

FIgURE 4: Plots of $\left|\beta_{x x x}\right|$ and $\left|\beta_{y y y}\right|$ versus $V_{0}$ with $\xi=0.001$ a.u. at two off-center dopant locations: (i) for $\left|\beta_{x x x}\right|$ at $r_{0}=42.43$ a.u., (ii) for $\left|\beta_{x x x}\right|$ at $r_{0}=70.71$ a.u., (iii) for $\left|\beta_{y y y}\right|$ at $r_{0}=42.43$ a.u., and (iv) for $\left|\beta_{y y y}\right|$ at $r_{0}=70.71$ a.u.

dopant location $\left(r_{0}=70.71\right.$ a.u. $)$ owing to a diminished dotimpurity interaction.

A similar saturation behavior is also envisaged in $\left|\beta_{x x x}\right|$ and $\left|\beta_{y y y}\right|$ profiles as a function of $V_{0}$ (Figure 4). Since the $|\beta|$ components assume zero value at on-center dopant location the figure depicts their profiles at near off-center $\left(r_{0}=42.43\right.$ a.u. $)$ and far off-center $\left(r_{0}=70.71\right.$ a.u. $)$ dopant locations. Here, also we notice a lower magnitude of $\left|\beta_{y y y}\right|$ component in comparison to its counterpart along $x$ direction. Interestingly, each of the $|\beta|$ component exhibits greater magnitude in the far off-center dopant location in comparison to that of near off-center position. The behavior happens to be quite obvious as already we have come across position dependent maximization in $|\beta|$ components.

3.2.3. Influence of Impurity Spread. We now turn our attention towards inspecting the influence of spatial stretch of impurity $(\xi)$ on the polarizability components for on and two off-center $\left(r_{0}=42.43\right.$ a.u. and 70.71 a.u. $)$ dopant locations (Figure 5). The $\alpha_{x x}$ and $\alpha_{y y}$ components initially delineate sort of decrease with increase in $\xi$ (in small $\xi$ domain) but ultimately culminate in some steady value with further increase in $\xi$ value. A small $\xi$ value implies that the impurity potential is diffused over a long spatial region, so also its influence. In the very low $\xi$ regime, this extreme diffusive nature of the impurity domain results in substantial dot-impurity interaction and consequently a large dispersive nature of the system. The polarizability components thus register somewhat large value. The scenario changes completely in the high $\xi$ regime. Now, a spatially quenched impurity potential undergoes very weak overlap with the dot confinement center resulting in small dispersive nature of the system. This causes a fall in the linear polarizability values. At very high value of $\xi$, the spatial stretch of impurity becomes highly condensed and the decreasing dispersive 


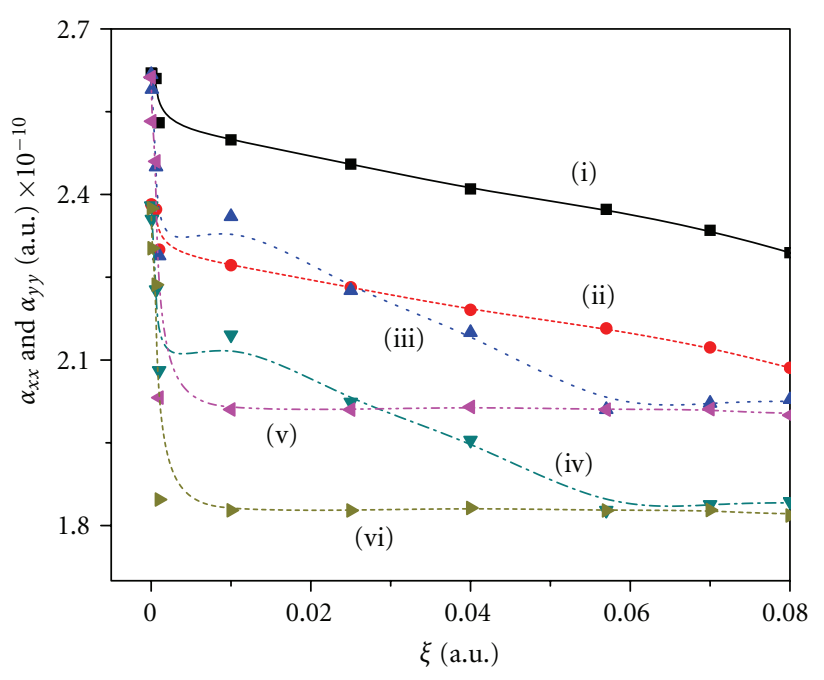

Figure 5: Plots of $\alpha_{x x}$ and $\alpha_{y y}$ versus $\xi$ with $V_{0}=1.0 \times 10^{-6}$ a.u. at on and two off-center dopant locations: (i) for $\alpha_{x x}$ at $r_{0}=0.0$ a.u., (ii) for $\alpha_{y y}$ at $r_{0}=0.0$ a.u., (iii) for $\alpha_{x x}$ at $r_{0}=42.43$ a.u., (iv) for $\alpha_{y y}$ at $r_{0}=42.43$ a.u., (v) for $\alpha_{x x}$ at $r_{0}=70.71$ a.u., and (vi) for $\alpha_{y y}$ at $r_{0}=70.71$ a.u.

nature of the system reaches its limit. The outcome being the steady values of the linear polarizability components. Also, as before, the $\alpha_{y y}$ components are smaller in magnitude in comparison to their $x$-directional counterpart. Furthermore, for each components, the extent of fall in its magnitude with increase in $\xi$ becomes progressively more pronounced with shift of the dopant from on- to off-center location. This is simply because of the fact that since a high $\xi$ value makes dot-impurity overlap already weak, a shift of dopant to more off-center location further aggravates the situation and hastens the fall of polarizability. Peculiarly, for the intermediate off-center impurity positions, the curve is not monotonous. It seems difficult to understand why in this case we do not encounter an otherwise straightforward curve. It appears that at intermediate dopant locations, along with dot-impurity overlap, the extent of dot confinement also plays a role in shaping the polarizability components. At these locations, a reduced dot-impurity overlap decreases the polarizability values. On the other hand, because of considerable dot-impurity separation the dot confinement also becomes less stringent making the system more flexible which enhances the polarizability values. The competitive behavior between these two opposite influences could be the cause behind the departure of the aforesaid profile from an otherwise monotonous plot.

$\left|\beta_{x x x}\right|$ and $\left|\beta_{y y y}\right|$ components, on the other hand, exhibit prominent maximization as a function of $\xi$ (Figure 6). Here, we present the corresponding profiles at near off-center $\left(r_{0}=42.43\right.$ a.u. $)$ and far off-center $\left(r_{0}=70.71\right.$ a.u. $)$ dopant locations and as usual the $|\beta|$ components assume zero value at on-center dopant location. The continual decrease in dopant's spatial stretch with increase in $\xi$ value might have some contrasting consequences on $|\beta|$ components. Although it reduces the confines of dot potential on

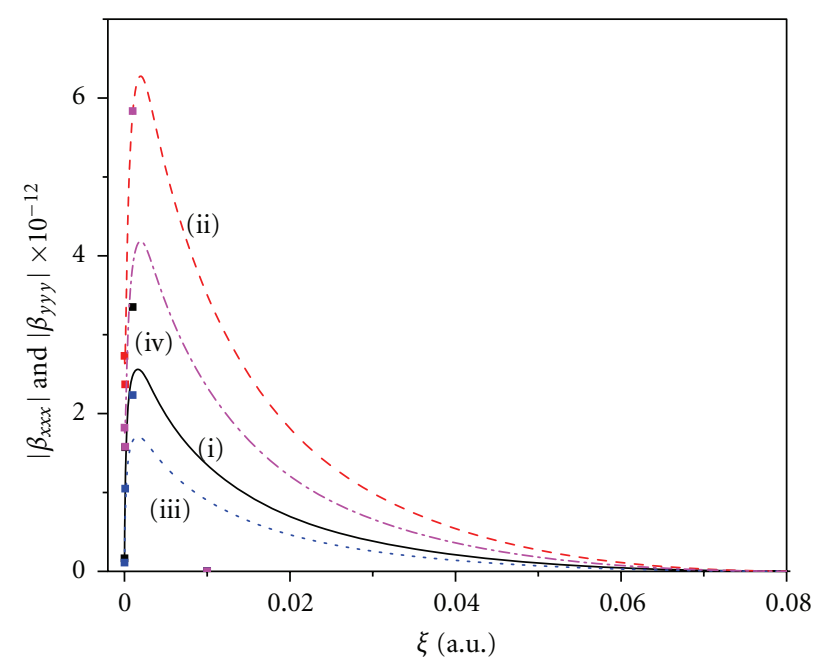

Figure 6: Plots of $\left|\beta_{x x x}\right|$ and $\left|\beta_{y y y}\right|$ versus $\xi$ with $V_{0}=1.0 \times$ $10^{-6}$ a.u. at two off-center dopant locations: (i) for $\left|\beta_{x x x}\right|$ at $r_{0}=$ 42.43 a.u., (ii) for $\left|\beta_{x x x}\right|$ at $r_{0}=70.71$ a.u., (iii) for $\left|\beta_{y y y}\right|$ at $r_{0}=$ 42.43 a.u., and (iv) for $\left|\beta_{y y y}\right|$ at $r_{0}=70.71$ a.u.

the dopant but simultaneously the dot-impurity repulsive interaction is also diminished. We feel that a changeover in the mutual dominance of these two contrasting factors could give rise to maximization of $\left|\beta_{y y y}\right|$ components at some particular $\xi$ value. As expected, here also we notice a lower magnitude of $\left|\beta_{y y y}\right|$ component in comparison to its counterpart along $x$-direction. Interestingly, each of the $|\beta|$ component exhibits greater magnitude in the far offcenter dopant location in comparison to that of near offcenter position. The behavior happens to be quite obvious as already we have visualized position dependent maximization in $|\beta|$ components.

\section{Conclusions}

The diagonal components of linear and first nonlinear polarizabilities of repulsive impurity doped quantum dots subject to a static electric field reveal intriguing features. For an in-depth analysis, we examine the roles played by impurity spread, impurity strength and most importantly impurity location meticulously on these components. We have found that the linear polarizability components decrease with increase in separation of dopant location from that of dot confinement center and also with an increase in spatial shrinkage of dopant potential. On the other hand, we envisage an increase in the said components with impurity strength. However, in all cases finally some steady behavior has been observed and $y$ direction has been found to be under more stringent confinement than the $x$ direction. The first nonlinear polarizability components evince maximization at some particular dopant location and for some particular value of spatial spread of dopant in absolute sense. The maximization appears to be due to the conflict between two opposing factors that foment and hinder the effective 
confinement of the dot on the dopant. However, as a function of dopant strength, we observe a persistent increase in $|\beta|$ values in absolute sense which culminates in a steady magnitude. We expect that the results obtained could have important implications in optical applications of quantum dot nanodevices.

At the fag end of the discussion, it appears to be quite significant to highlight the new findings in the present investigation in the light of the results of [23]. First of all, we want to mention that in the present study we have explored the role of impurity location rigorously which was absent from our earlier study on frequency-dependent polarizability (FDP) [23]. Secondly, in [23], we observed that in the limitingly small dopant strength domain an increase in dopant strength causes an initial lull in the FDP values. However, as the dopant strength is increased as bit FDP values begin to increase smoothly with dopant strength culminating in a steady value. In the present study, although we have found some kind of similar trend at high $V_{0}$ limit, but behavior is absolutely different in small $V_{0}$ domain. The role of dopant's spatial spread comes out to be quite distinct in the two investigations. In the earlier study, we found that squeezing the spatial expansion of the dopant domain results in persistent increase in FDP values whereas in the present enquiry a reverse behavior has been envisaged. We hope to explore a rigorous investigation on the frequency-dependent and static polarizability components on a comparative basis in near future.

In the present investigation, we did not consider the influence of size on the optical properties. Although in principle the dot wave function can stretch up to infinity but in practice, it actually terminates at some finite values. Thus, the size effect would be important at length scales within the actual termination of wave function.

It is quite expected that donor and acceptor impurity would exhibit distinct impacts on the NLO properties. Recently, Hazra et al. have investigated the role of donor and acceptor impurities in a slightly different context. It needs further study to precisely understand the distinct roles of acceptor and donor impurity pertinent to the present investigation.

\section{Acknowledgment}

The authors N. K. Datta and M. Ghosh thank D. S. T.-F. I. S. T (Government of India) and U. G. C.-S. A. P (Government of India) for financial support.

\section{References}

[1] P. M. Koenraad and M. E. Flatté, "Single dopants in semiconductors," Nature Materials, vol. 10, pp. 91-100, 2011.

[2] H. J. Queisser and E. E. Haller, "Defects in semiconductors: some fatal, some vital," Science, vol. 281, no. 5379, pp. 945950, 1998

[3] B. Çakir, Y. Yakar, A. Özmen, M. Ö. Sezer, and M. Şahin, "Linear and nonlinear optical absorption coefficients and binding energy of a spherical quantum dot," Superlattices and Microstructures, vol. 47, no. 4, pp. 556-566, 2010.
[4] Y. Yakar, B. Çakir, and A. Özmen, "Calculation of linear and nonlinear optical absorption coefficients of a spherical quantum dot with parabolic potential," Optics Communications, vol. 283, no. 9, pp. 1795-1800, 2010.

[5] J. L. Movilla and J. Planelles, "Off-centering of hydrogenic impurities in quantum dots," Physical Review B, vol. 71, no. 7, Article ID 075319, pp. 1-7, 2005.

[6] M. J. Kelly, Low-Dimensional Semiconductors, Oxford University Press, Oxford, UK, 1995.

[7] C. P. Poole Jr and F. J. Owens, Introduction to Nanotechnology, Wiley, New York, NY, USA, 2003.

[8] J. L. Brédas, C. Adant, P. Tackx, A. Persoons, and B. M. Pierce, "Third-order nonlinear optical response in organic materials: theoretical and experimental aspects," Chemical Reviews, vol. 94, no. 1, pp. 243-278, 1994.

[9] P. N. Prasad and D. J. Williams, Introduction to Non-linear Optical Effects in Molecules and Polymers, Wiley, New York, NY, USA, 1991.

[10] G. I. Stegemen, A. Miller, and J. E. Midwinter, Eds., Photonics in Switching volume 1 Background and Components, Academic Press, Boston, Mass, USA, 1993.

[11] D. S. Chemla and J. Zyss, Eds., Non-linear Optical Properties of Molecules and Crystals, vol. 1, Academic Press, New York, NY, USA, 1983.

[12] A. Leclercq, E. Zojer, S. H. Jang et al., "Quantumchemical investigation of second-order nonlinear optical chromophores: comparison of strong nitrile-based acceptor end groups and role of auxiliary donors and acceptors," Journal of Chemical Physics, vol. 124, no. 4, Article ID 044510, pp. 1-7, 2006.

[13] Z.-Y. Hu, A. Fort, M. Barzoukas, A. K. Y. Jen, S. Barlow, and S. R. Marder, "Trends in optical nonlinearity and thermal stability in electrooptic chromophores based upon the 3(dicyanomethylene)-2,3-dihydrobenzothiophene-1, 1-dioxide acceptor," Journal of Physical Chemistry B, vol. 108, no. 25, pp. 8626-8630, 2004.

[14] G. Ramos-Ortiz, M. Cha, S. Thayumanavan, J. C. Mendez, S. R. Marder, and B. Kippelen, "Third-order optical autocorrelator for time-domain operation at telecommunication wavelengths," Applied Physics Letters, vol. 85, no. 2, pp. 179 $181,2004$.

[15] G. J. Ashwell, R. C. Hargreaves, C. E. Baldwin, G. S. Bahra, and C. R. Brown, "Improved second-harmonic generation from Langmuir-Blodgett films of hemicyanine dyes," Nature, vol. 357, no. 6377, pp. 393-395, 1992.

[16] S. R. Marder, J. E. Sohn, and G. D. Stucky, Eds., Materials for Non-Linear Optics: Chemical Perspectives, ACS Sympos, vol. 455, American Chemical Society, Washington, DC, USA, 1991.

[17] R. Sen, D. Majumdar, S. P. Bhattacharyya, and S. N. Bhattacharyya, "Modeling hyperpolarizabilities of some TICT molecules and their analogues," Journal of Physical Chemistry, vol. 97, no. 29, pp. 7491-7498, 1993.

[18] A. D. Buckingham, E. P. Concannon, and I. D. Hands, "Hyperpolarizability of interacting atoms," Journal of Physical Chemistry, vol. 98, no. 41, pp. 10455-10459, 1994.

[19] K. L. C. Hunt and A. D. Buckingham, "The polarizability of H2 in the triplet state," The Journal of Chemical Physics, vol. 72, no. 4, pp. 2832-2840, 1980.

[20] F. Furtmayr, M. Vielemeyer, M. Stutzmann, A. Laufer, B. K. Meyer, and M. Eickhoff, "Optical properties of Si- and Mgdoped gallium nitride nanowires grown by plasma-assisted molecular beam epitaxy," Journal of Applied Physics, vol. 104, no. 7, Article ID 074309, 2008. 
[21] O. Weidemann, P. K. Kandaswamy, E. Monroy, G. Jegert, M. Stutzmann, and M. Eickhoff, "GaN quantum dots as optical transducers for chemical sensors," Applied Physics Letters, vol. 94, no. 11, Article ID 113108, 2009.

[22] K. Sarkar, N. Kumar Datta, and M. Ghosh, "Frequency dependent linear and non-linear response properties of electron impurity doped quantum dots: influence of impurity location," Physica E, vol. 42, no. 5, pp. 1659-1666, 2010.

[23] N. K. Datta and M. Ghosh, "Impurity strength and impurity domain modulated frequency-dependent linear and second non-linear response properties of doped quantum dots," Physica Status Solidi B, vol. 248, no. 8, pp. 1941-1948, 2011.

[24] V. Halonen, P. Hyvönen, P. Pietiläinen, and T. Chakraborty, "Effects of scattering centers on the energy spectrum of a quantum dot," Physical Review B, vol. 53, no. 11, pp. 69716974, 1996.

[25] V. Halonen, P. Pietilinen, and T. Chakraborty, "Opticalabsorption spectra of quantum dots and rings with a repulsive scattering centre," Europhysics Letters, vol. 33, no. 5, pp. 377382, 1996.

[26] R. Turton, The Quantum Dot. A Journey into Future Microelectronics, Oxford University Press, New York, NY, USA, 1995.

[27] L. Jacak, P. Hawrylak, and A. Wojos, Quantum Dots, SpringerVerlag, Berlin, Germany, 1998.

[28] M. A. Reed, Quantum Dots, Scientific American, Ocala, Fla, USA, 1993.

[29] Y. Alhassid, "The statistical theory of quantum dots," Reviews of Modern Physics, vol. 72, no. 4, pp. 895-968, 2000.

[30] J. Adamowski, A. Kwaśniowski, and B. Szafran, "LO-phononinduced screening of electron-electron interaction in Dcentres and quantum dots," Journal of Physics Condensed Matter, vol. 17, no. 28, pp. 4489-4500, 2005.

[31] S. Bednarek, B. Szafran, K. Lis, and J. Adamowski, "Modeling of electronic properties of electrostatic quantum dots," Physical Review B, vol. 68, no. 15, Article ID 155333, 9 pages, 2003.

[32] P. D. Siverns, S. Malik, G. McPherson et al., "Scanning transmission-electron microscopy study of InAs/GaAs quantum dots," Physical Review B, vol. 58, no. 16, pp. R10127R10130, 1998.

[33] S. Bednarek, B. Szafran, and J. Adamowski, "Theoretical description of electronic properties of vertical gated quantum dots," Physical Review B, vol. 64, no. 19, Article ID 195303, pp. 1953031-19530313, 2001.

[34] A. P. Alivisatos, "Perspectives on the physical chemistry of semiconductor nanocrystals," Journal of Physical Chemistry, vol. 100, no. 31, pp. 13226-13239, 1996.

[35] A. A. Guzelian, U. Banin, A. V. Kadavanich, X. Peng, and A. P. Alivisatos, "Colloidal chemical synthesis and characterization of InAs nanocrystal quantum dots," Applied Physics Letters, vol. 69 , no. 10 , pp. 1432-1434, 1996.

[36] I. S. Gradshteyn and I. M. Ryzhik, Tables of Integrals, Series, and Products, Corrected and Enlarged Edition, Academic Press, City, State, USA, 1980.

[37] J. J. P. Stewart and K. M. Dieter, "Calculation of the nonlinear optical properties of molecules," Journal of Computational Chemistry, vol. 11, no. 1, pp. 82-87, 1990.

[38] P. Dutta and S. P. Bhattacharyya, "On exact calculation of response properties of oscillators in static electric field: a Fourier grid Hamiltonian approach. I. One-dimensional systems," International Journal of Quantum Chemistry, vol. 51, no. 5, pp. 293-305, 1994.
[39] G. Maroulis and A. J. Thakkar, "Multipole moments, polarizabilities, and hyperpolarizabilities for N2 from fourth-order many-body perturbation theory calculations," The Journal of Chemical Physics, vol. 88, no. 12, pp. 7623-7632, 1988.

[40] G. Maroulis, "Hyperpolarizability of H2O," The Journal of Chemical Physics, vol. 94, no. 2, pp. 1182-1190, 1991.

[41] G. Maroulis, "Hyperpolarizability of $\mathrm{H}_{2} \mathrm{O}$," Journal of Chemical Physics, vol. 94, pp. 1182-1190, 1991.

[42] T. Chakraborty, Quantum Dots-A Survey of the Properties of Artificial Atoms, Elsevier, Amsterdam, The Netherlands, 1999.

[43] S. V. Nistor, M. Stefan, L. C. Nistor, E. Goovaerts, and G. Van Tendeloo, "Incorporation and localization of substitutional Mn2+ ions in cubic ZnS quantum dots," Physical Review B, vol. 81, no. 3, Article ID 035336, 2010.

[44] P. A. Sundqvist, V. Narayan, S. Stafström, and M. Willander, "Self-consistent drift-diffusion model of nanoscale impurity profiles in semiconductor layers, quantum wires, and quantum dots," Physical Review B, vol. 67, no. 16, Article ID 165330, pp. 1653301-1653308, 2003. 

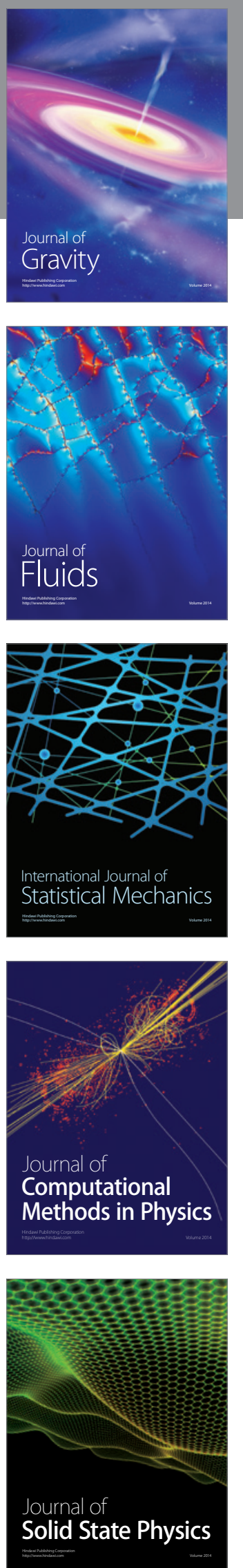
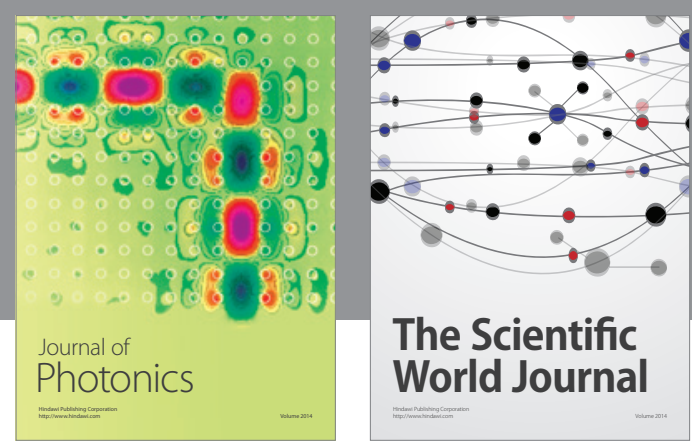

The Scientific World Journal

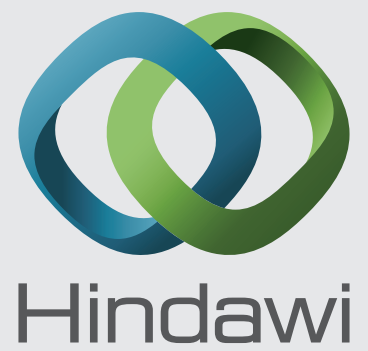

Submit your manuscripts at http://www.hindawi.com
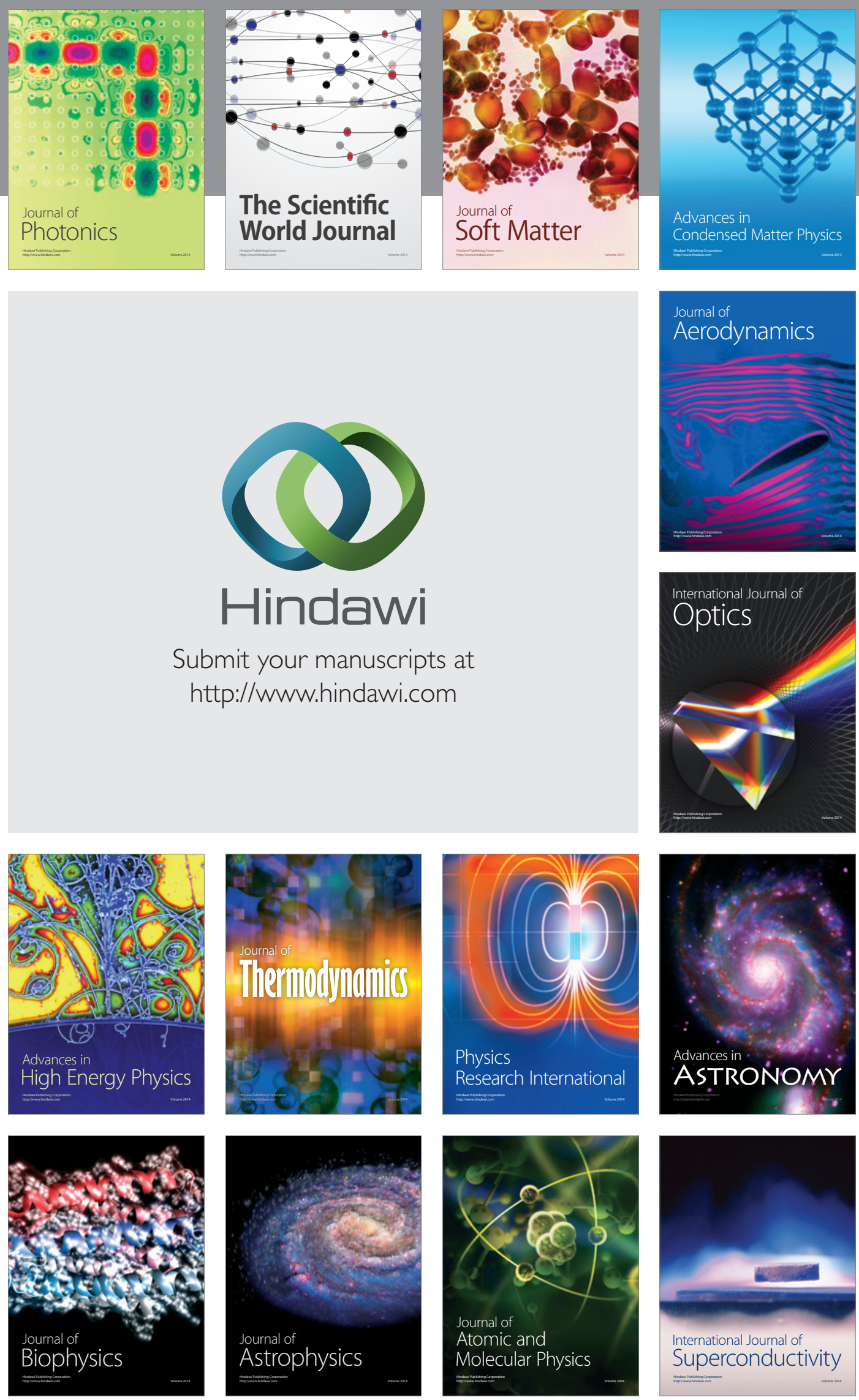
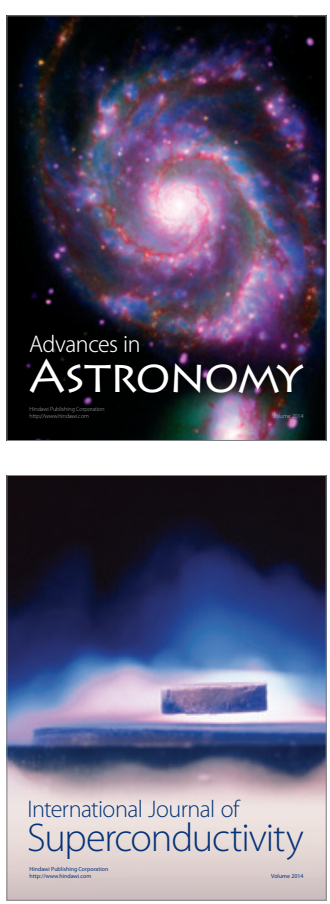